Observations of rotation in JET plasmas with electron heating by ion cyclotron resonance heating

This article has been downloaded from IOPscience. Please scroll down to see the full text article.

2012 Plasma Phys. Control. Fusion 54074007

(http://iopscience.iop.org/0741-3335/54/7/074007)

View the table of contents for this issue, or go to the journal homepage for more

Download details:

IP Address: 193.190.204.60

The article was downloaded on 11/07/2012 at 14:08

Please note that terms and conditions apply. 


\title{
Observations of rotation in JET plasmas with electron heating by ion cyclotron resonance heating
}

\author{
T Hellsten ${ }^{1}$, T J Johnson ${ }^{1}$, D Van Eester ${ }^{2}$, E Lerche², Y Lin ${ }^{4}$, M-L Mayoral ${ }^{3}$, \\ J Ongena $^{2}$, G Calabro ${ }^{9}$, K Crombé ${ }^{2}$, D Frigione ${ }^{9}$, C Giroud ${ }^{3}$, M Lennholm ${ }^{3}$, \\ P Mantica $^{5}$, M F F Nave ${ }^{6}$, V Naulin ${ }^{8}$, C Sozzi $^{9}$, W Studholme ${ }^{3}$, T Tala ${ }^{7}$, \\ T Versloot $^{10}$ and JET-EFDA Contributors ${ }^{11}$ \\ JET-EFDA, Culham Science Centre, OX14 3DB, Abingdon, UK \\ ${ }^{1}$ Euratom-VR Association, EES, KTH, Stockholm, Sweden \\ ${ }^{2}$ LPP-ERM/KMS, Association Euratom-Belgian State, 1000 Brussels, Belgium, TEC Partner \\ ${ }^{3}$ Euratom-CCFE Fusion Association Culham Science Centre, UK \\ ${ }^{4}$ Plasma Science and Fusion Centre, MIT, Cambridge, USA \\ ${ }^{5}$ Instituto di Fisica del Plasma, EURATOM-ENEA-CNR Association, Milan, Italy \\ ${ }^{6}$ Euratom/IST Fusion Association, Instituto de Plasmas e Fusão Nuclear, Instituto Superior Technico, Universidade \\ Technica de Lisboa, Portugal \\ ${ }^{7}$ HUT, Association Euratom-Tekes, Finland \\ ${ }^{8}$ Association Euratom-Ris $\varnothing$, National Laboratory, Denmark \\ ${ }^{9}$ EURATOM-ENEA sulla Fusione, C. R. Frascati, Frascati, Italy \\ ${ }^{10}$ FOM Institute for Plasma Physics Rijnhuizen, Association EURATOM-FOM, The Netherlands, TEC Partner
}

Received 14 October 2011, in final form 3 December 2011

Published 20 June 2012

Online at stacks.iop.org/PPCF/54/074007

\begin{abstract}
The rotation of L-mode plasmas in the JET tokamak heated by waves in the ion cyclotron range of frequencies (ICRF) damped on electrons, is reported. The plasma in the core is found to rotate in the counter-current direction with a high shear and in the outer part of the plasma with an almost constant angular rotation. The core rotation is stronger in magnitude than observed for scenarios with dominating ion cyclotron absorption. Two scenarios are considered: the inverted mode conversion scenarios and heating at the second harmonic ${ }^{3} \mathrm{He}$ cyclotron resonance in $\mathrm{H}$ plasmas. In the latter case, electron absorption of the fast magnetosonic wave by transit time magnetic pumping and electron Landau damping (TTMP/ELD) is the dominating absorption mechanism. Inverted mode conversion is done in $\left({ }^{3} \mathrm{He}\right)-\mathrm{H}$ plasmas where the mode converted waves are essentially absorbed by electron Landau damping. Similar rotation profiles are seen when heating at the second harmonic cyclotron frequency of ${ }^{3} \mathrm{He}$ and with mode conversion at high concentrations of ${ }^{3} \mathrm{He}$. The magnitude of the counter-rotation is found to decrease with an increasing plasma current. The correlation of the rotation with the electron temperature is better than with coupled power, indicating that for these types of discharges the dominating mechanism for the rotation is related to indirect effects of electron heat transport, rather than to direct effects of ICRF heating. There is no conclusive evidence that mode conversion in itself affects rotation for these discharges.
\end{abstract}

(Some figures may appear in colour only in the online journal)

\section{Introduction}

The rotation of plasmas is considered to be beneficial for confinement and stability by suppressing drift waves and

11 See appendix of Romanelli F et al 2010 Proc. 23rd Int. Conf. on Fusion Energy 2010 (Deajeon, Korea) (IAEA: Vienna). resistive wall modes. The main source for driving rotation in present day experiments is neutral beam injection. However, the momentum input by neutral beam injection in ITER and future thermonuclear reactors will be rather low due to the high density and large volume of ITER plasmas, thus rendering the penetration of the beams into the core of the 
plasma difficult. It is therefore of interest to investigate alternative methods to generate rotation. Toroidal rotation of the plasma is often seen during ion cyclotron resonance heating (ICRH) even when the momentum inputs by the waves are negligible [1-13]. In general, the rotation is rather small and caused by several weak mechanisms that are not fully understood. Some of them are specific to the heating method, caused by absorption of the momentum of the waves used for heating or by finite drift orbit effects of energetic ions produced by heating. Others are caused by changes in the plasma temperature, and are not specific to the heating method (intrinsic rotation). Some effects have been experimentally confirmed, but there still remain other observations which need explaining. The strongest observed counter-rotation with ICRH alone in JET is obtained with a strong magnetic field ripple $\delta=\left(B_{\max }-B_{\min }\right) /\left(B_{\max }+B_{\min }\right) \sim 1.5 \%$ [8], where $B_{\max }$ and $B_{\min }$ are the maximum and minimum field at the plasma boundary, at the outer midplane. Plasma rotation has also been observed during ohmic heating [14-16] and in experiments using waves in the electron cyclotron range of frequencies (ECRF) [17-19].

The main differences between ICRF and ECRF are (i) the ratio between momentum and energy densities for ECRF waves is smaller than for ICRF waves; and (ii) ECRF waves are directly absorbed on electrons.

The rotation profiles of ECRH and ohmic heated plasmas have some similar common characteristics with those found in JET with ICRH, in particular with those with direct electron heating in the absence of net momentum input. In TCV centrally peaked counter-rotating profiles at a low plasma current and a low density were found in limiter discharges, even without ECRH, which at a sufficiently high plasma current changed to a co-current rotation, when the plasma density exceeded a well-defined threshold $[16,17]$. Divertor plasma had similar behaviour, but changed from a co-current rotation to a counter-current rotation [17], in contrast to JET where the JET observations were more in line with the observations of the limiter discharges in TCV, where a co-current rotation was seen at high plasma currents and a counter-current rotation at low plasma currents. In a later study it was shown that the up-down asymmetry of the magnetic flux surfaces strongly influenced the rotation; it was expected to be the mechanism breaking the symmetry, and causing net momentum transport [18]. From a multi-machine comparison it was concluded that the countercurrent intrinsic rotation was produced in regimes where the logarithmic density gradient was sufficiently large and the turbulence regime was either in the trapped electron mode (TEM) or in transition between the TEM and ion temperature gradient (ITG) regime [19]. In TCV it was also observed that the peaked rotation profiles during ECRH were flattened by sawtooth crashes [15].

Various mechanisms have been identified to give rise to torques in plasmas heated with ICRH, for which the estimated rotation profiles caused by the torque are comparable to those observed in experiments. The momentum input by directed waves has been predicted to affect the rotation, and evidence of this has been obtained previously by comparing plasmas heated with fast magnetosonic waves propagating in the co- and the counter-current directions with modelling [13]; the absorbed wave momentum and the change in the toroidal rotation was in agreement with simulations with the SELFO code [20,21]. However, there are changes in the rotation profiles that cannot adequately be modelled with existing heating codes. As ions are heated from thermal to suprathermal energies, finite orbit width effects can give rise to rotation. In recent experiments, with $\mathrm{H}$-minority heating in deuterium plasmas, it was shown that the torque caused by these suprathermal ions was not sufficient to fully explain the rotation; assuming that the momentum confinement was similar to the energy confinement time, the rotation resulting from the finite orbit effect was about a factor of three too low [3]. In a spatial dispersive medium, the change in the momentum of the waves, by non-resonant interactions as the waves propagate across the plasma, can give rise to a torque in the plasma $[22,23]$. As the wave is absorbed, the momentum is transferred to the plasma. This change in momentum by non-resonant interactions results in a torque on the plasma with a dipolar characteristic and adds to the torque from the momentum the wave had when it was launched at the antenna. The change in momentum is particularly important for slow waves like those obtained by a mode conversion of the fast magnetosonic wave, and could therefore result in large torques on the plasma in mode conversion experiments. The emission and absorption of drift waves, causing anomalous transport, can in a similar way give rise to dipolar-like torques due to the universal nature of the momentum and energy changes in wave-particle interactions.

The launched fast magnetosonic ICRF waves can be absorbed by ions and electrons either directly or indirectly through mode conversion to slow waves. Ion cyclotron damping at lower harmonics is often the dominating damping process of ICRH. If the power density is high, high-energy tails develop in the resonant ion distribution functions, and a large fraction of the power absorbed by ions will then be transferred to the electrons. High-energy ions with broad orbits, created by ICRH, can give rise to radial currents driving the rotation. Most of the earlier rotation studies on JET $[3,8]$ with ICRH were done in scenarios with a dominating ion cyclotron resonance absorption, where the power was transferred to ions and electrons by collisions. Few studies were done with direct electron absorption of the fast magnetosonic waves by transit time magnetic pumping and electron Landau damping (TTMP/ELD), which in current experiments is often a weak damping mechanism. Another way to heat electrons without first heating ions is to convert the magnetosonic waves into slow kinetic waves damped by ELD or ion cyclotron absorption. The electron damping of the mode converted wave is usually strong, and dominates the absorption above a certain minority concentration. It is usually more localized than the power deposition by a direct absorption of the fast magnetosonic wave by TTMP/ELD.

Most rotation studies in JET have been done in ohmically heated and L-mode plasmas. The typical rotation profiles observed during $\mathrm{H}$-minority central ICRF heating in the absence of a strong field ripple $(\delta<0.08 \%)$ in JET, show a positive rotation (i.e. in the direction of the plasma current) for most of the plasma radius with little shear in the outer part of 
the plasma. In the core, rotation can nevertheless be positive or negative [3] depending on the plasma current. For low plasma currents the rotation profiles are often hollow in the centre; in some cases even with a negative rotation in the plasma centre. In contrast, for large plasma currents, the rotation profiles are positive and sometimes even peaked at the centre. In earlier JET experiments, the central toroidal rotation profiles were modified by varying the relative phasing of the antenna straps in the individual A2 antenna modules and the position of the ion cyclotron resonance [7]. In these experiments the best overall scaling of the rotation in the core was obtained with respect to plasma current over line-averaged central electron density, as shown in [3]. However, the scattering of the data was large indicating variations due to additional variables.

That the rotation depends on the confinement in JET was reported in $[2,24]$, both co- and counter-rotation were observed in $\mathrm{H}$ - and L-modes. A detailed study on how the rotation profiles depend on the confinement properties of the discharge in Alcator C-Mod with H-minority heating, was reported in [4]. Flat central co-rotation profiles were found in EDA (enhanced $\mathrm{D}_{\alpha}$ ) H-mode plasmas [25]; peaked co-rotation profiles were found in ELM free H-modes and hollow ones in discharges with internal transport barriers, where ELM stands for edge localized mode. Time dependent measurements of the rotation profiles in $\mathrm{H}$-modes demonstrated that rotation in the positive direction propagates inwards from the edge. It has been suggested that this propagation is anomalously fast and somewhat slower than that of the energy confinement. In L-mode both co- and counter-rotation was observed, although mostly rotated in the counter-direction. Another interesting observation of L-mode plasmas in Alcator C-Mod was that the rotation in the outer part of the plasma was sensitive to the magnetic field geometry [9]. Discharges limited to the inner wall or with a lower single null had a counter-rotation, whereas discharges with an upper single null demonstrated a co-rotation. Recently, mode conversion experiments in Alcator C-Mod demonstrated a relatively large co-current rotation in the core in a narrow parameter window [9].

The scaling of toroidal rotation in JET, proportional to the plasma current divided with line-averaged density reported in [3], may seem to differ fundamentally from Alcator C-Mod where rotation scales with the ratio of diamagnetic energy over the plasma current derived from discharges both in Land H-modes [10-12]. Note in the previously mentioned JET experiments [3] at low plasma currents, which were in L-mode, not only the rotation profiles were hollow, but the difference in rotation between edge and core increased in magnitude with a decreasing current, which is more in line with the Alcator C-Mod scaling. However, at high plasma currents the core rotation was positive and the edge-core difference increased with the plasma current in contrast to the Alcator C-Mod scaling. These observations seem to indicate that rotation in the core and outer part of the plasma are driven by different mechanisms and a better approach could be to look for a scaling with respect to the difference in rotation between the edge and the core.

In order to improve the understanding of the effect of ICRF waves on rotation, dedicated experiments were performed on JET using two ICRF heating schemes with direct electron heating without generating fast ions: (i) ICRF mode conversion in $\left({ }^{3} \mathrm{He}\right)-\mathrm{H}$ plasmas and (ii) ICRF heating at the second harmonic ${ }^{3} \mathrm{He}$ cyclotron resonance. Comparisons between different ICRF heating methods in similar plasmas, i.e. the same plasma current, magnetic field, plasma density and temperature, demonstrate that electron heating gives rise to a counter-torque. The rotation profiles are similar as those obtained in experiments from other machines with ECRH and ohmic heating, indicating that the anomalous electron transport by drift waves gives rise to a counter-torque.

In this paper, the rotation measurement for the two cases will be carefully reviewed. The experimental setup is presented in section 2, the experimental results are presented in section 3 and conclusions with discussions are given in section 4 .

\section{Experimental setup}

The experiments described in this paper were carried out with hydrogen plasmas in L-mode. ${ }^{3} \mathrm{He}$ was injected with feedbackcontrolled gas puffing, using the method described in [26, 27]. The ${ }^{3} \mathrm{He}$ concentration was estimated from the intensity of emission lines of the various plasma constituents in the divertor with visible spectroscopy. The plasmas were heated with the JET ICRF system using the four so-called A2 antennas. Each antenna has four straps and is equipped with a Faraday screen, which is nearly parallel with the magnetic field, in order to reduce the coupling to slow waves. The currents in the straps can be phased relative to each other in order to change the directivity of the launched waves. Dipole phasing (i.e. relative phasing of the four antenna straps: $0, \pi, 0, \pi$ ) producing a nearly symmetric wave spectrum was used to reduce the net angular momentum of the waves.

In the experiments using mode conversion, the toroidal magnetic field at the plasma centre (major radius $R_{0}=2.96 \mathrm{~m}$ ) was either 3.41 or $2.95 \mathrm{~T}$, the plasma current $I_{\mathrm{p}}=1.8 \mathrm{MA}$, the ICRF frequency $f=32 \mathrm{MHz}$ and the ${ }^{3} \mathrm{He}$ concentration varied between $1 \%$ and $17 \%$. In the experiment using second harmonic ${ }^{3} \mathrm{He}$ heating $B_{0}=2.65 \mathrm{~T}, I_{\mathrm{p}}=1.4 \mathrm{MA}$, the ICRF frequency $f=52 \mathrm{MHz}$ and the ${ }^{3} \mathrm{He}$ concentration varied from $5-25 \%$.

The main diagnostic for these experiments was the active charge exchange recombination spectroscopy (CXRS) providing rotation and temperature profiles of $\mathrm{C}^{6+}$, at 12 radial positions, with the innermost channel at a major radius $R=$ $2.88 \mathrm{~m}$ and the outermost channel at $R=3.78 \mathrm{~m}$. The CXRS diagnostic needs fast hydrogen or deuterium ions injected from the neutral beams for the charge exchange reaction with $\mathrm{C}^{6+}$, and therefore perturbs rotation measurements. D beams were used in the experiments reported here. Since the RF-induced rotation is weak, even one beam with $2 \mathrm{MW}$ power will significantly affect the rotation. To reduce the perturbation only the first $50 \mathrm{~ms}$ of short beam blips of $200 \mathrm{~ms}$ were used and a time interval of $0.5 \mathrm{~s}$ was left between the beam blips to relax the perturbation. The perturbation of the rotation by the beam blip can be assessed by comparing the plasma acceleration during the beam blip in $50 \mathrm{~ms}$ intervals [3]. The uncorrected rotation at the first $50 \mathrm{~ms}$ of the beam blip used in the graphs 

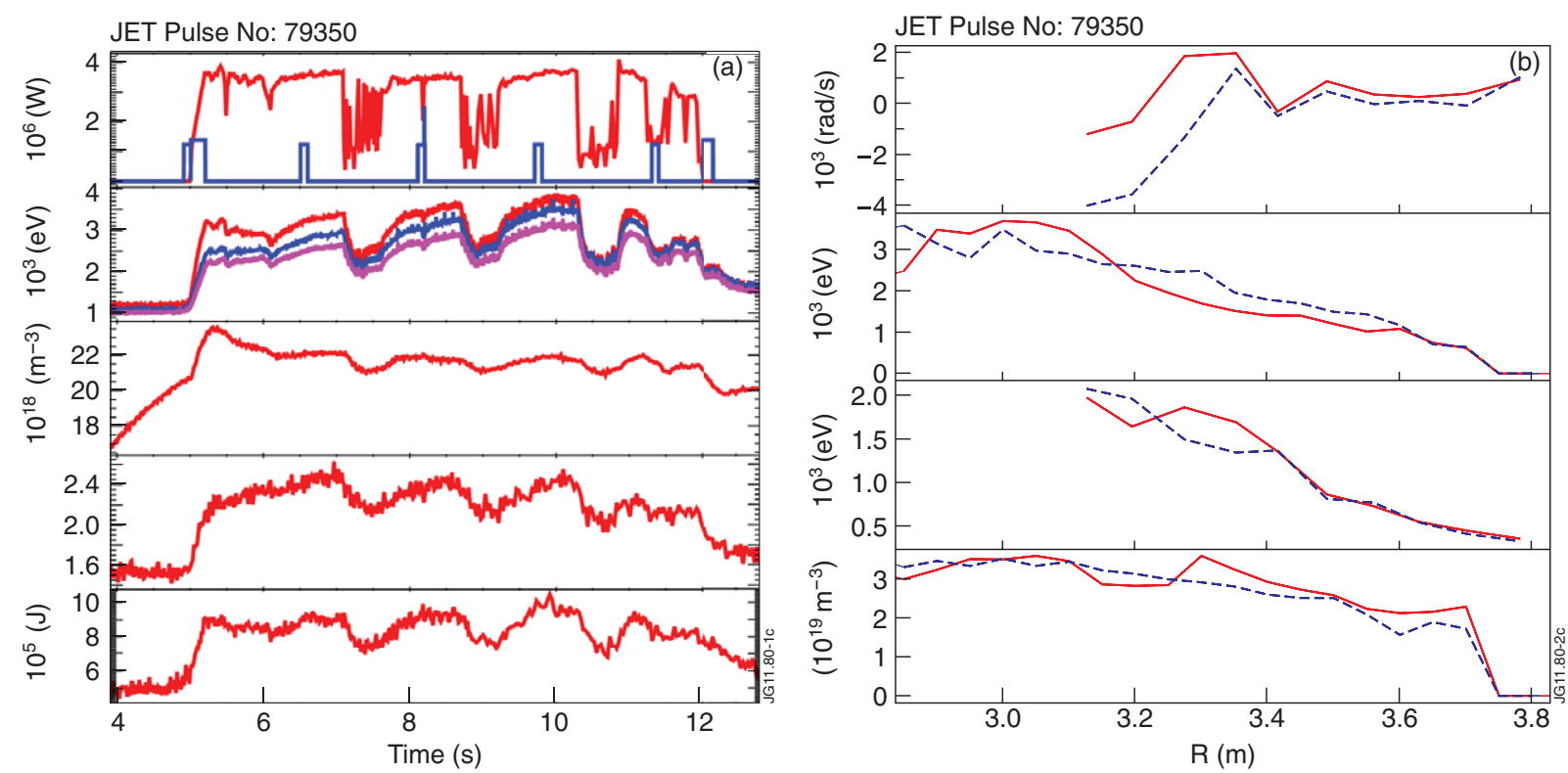

Figure 1. (a) Time traces of discharge \#79350. RF (red) and NBI (blue) power (top graph), electron temperature (second graph) measured with the ECE radiometer at three positions, $2.95 \mathrm{~m}$ (red), $2.75 \mathrm{~m}$ (blue) and $2.67 \mathrm{~m}$ (magenta). The line-averaged electron density (third graph), $Z_{\text {eff }}$ (fourth graph) and diamagnetic energy (bottom graph). (b) Profiles of toroidal angular rotation (top graph), ion temperature (second graph), electron temperature measured with LIDAR (third graph), electron density profiles measured with LIDAR (fourth graph) all at the time of the second and fifth beam blips at $t=6.51 \mathrm{~s}$ (red full) and $t=11.31 \mathrm{~s} \mathrm{(blue} \mathrm{dashed),} \mathrm{respectively.}$

is close to the corrected value. Because of the relatively low rotation velocity, the rotation measurements have large relative error bars, in the order of $50 \%$, making it difficult to draw conclusions when the changes are small. The error bars were the largest for the two innermost channels therefore they have been omitted in some of the graphs. The measurements after the application of ICRF heating showed less fluctuations. In those cases where the measured data were useable, the rotation in the outer part of the plasma was similar for the ohmic phase and the phase with ICRF heating.

The electron temperature is measured with a 96 channel ECE radiometer system [28] from the electron cyclotron emission. The line-integrated electron density was measured with the interferometer. The electron density and temperature profiles were measured with LIDAR. High-energy ions were detected using gamma emission (detected by a horizontal and vertical gamma camera), resulting from fast particle induced nuclear reactions in the plasma. In order to asses whether fast ions were produced by ICRH, energy spectra of fast neutrals leaving the plasma were measured with the neutral particle analyser (NPA).

The ion heating profiles could not be measured because of the perturbation in rotation, caused by the neutral beams prohibiting measurements of the ion power deposition, which would have required longer neutral beam pulses. The measured rotation profiles in the ohmic heating phase, before the onset of ICRF heating, were often unreliable due to having large spatial variations. Time traces of a typical pulse are shown in figure 1 $(a)$. The ICRF power includes modulation phases in order to determine the power deposition profiles to the electrons. The increase in the electron density and $Z_{\text {eff }}$ with ICRF power suggest that interaction at the antenna results in increased density containing a certain fraction of impurity ions, which is typical of these low absorption schemes [29]. Typical rotation, density and temperature profiles at the time of two beam blips are shown in figure $1(b)$.

\section{Experimental results}

The measured rotation in the outer part of the plasma, i.e. for major radii $R>3.4 \mathrm{~m}$, did not correlate with the ICRF power, but seemed to vary more between different sessions than within a session. These variations were more or less within the error bars, the causes have not yet been identified. Assuming that the measured rotation in the outer part of the plasma is not an instrumental offset, it has to be driven by a torque at the plasma edge, outside the first measurement points, since the angular momentum is often nearly constant in the radial range $3.4 \mathrm{~m}<R<3.78 \mathrm{~m}$.

\subsection{Inverted mode conversion in $\left({ }^{3} \mathrm{He}\right)-\mathrm{H}$ plasmas}

The power depositions for the inverted mode converted heating scenarios are expected to depend on the ICRH frequency, the magnetic field, the ratio between ${ }^{3} \mathrm{He}$ and $\mathrm{H}$ densities and impurities. Usually the mode converted waves are absorbed close to the mode conversion region. For mode conversion to take place the minority density has to exceed a certain threshold, which depends on the temperature and parallel wave number; below this threshold cyclotron damping takes place. Compared with standard mode conversion scenarios, the inverted scenarios have minority ion species with a smaller charge to mass ratio than the majority ion species. In these inverted mode conversion scenarios the fast wave (propagating inwards from the low-field side) first tunnels through the 


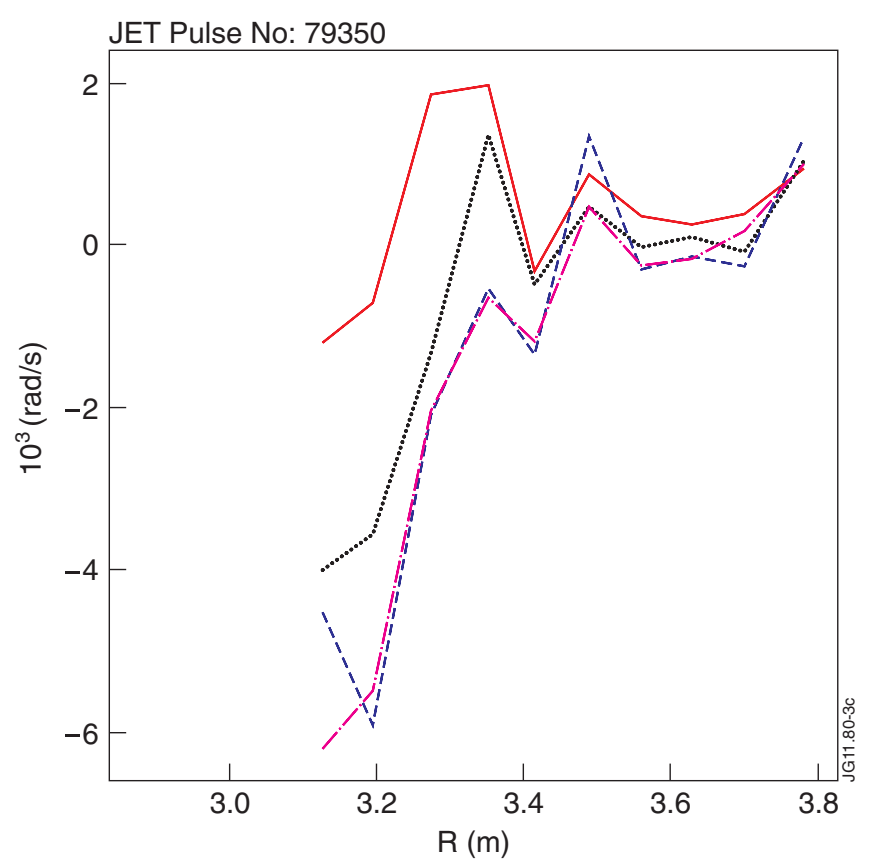

Figure 2. Toroidal angular rotation profiles for discharge \#79350, for which the ${ }^{3} \mathrm{He}$ concentration varied from $2-7 \%$, are shown in figure $1(a)$, at the time of the second beam blip at $t=6.51 \mathrm{~s}$ (red, full line), the third blip at $t=8.12 \mathrm{~s}$ (blue, dashed line), the forth blip at $t=9.72 \mathrm{~s}$ (magenta, dashed dotted line) and the fifth at $t=11.31 \mathrm{~s}$ (black, dotted line). The confidence interval is $\pm 0.5 \mathrm{krad} \mathrm{s}^{-1}$ in the outer part and $\pm 2 \mathrm{krad} \mathrm{s}^{-1}$ in the core.

right- and left-hand cut-off and then reaches the mode conversion layer where it converts to a kinetic Alfvén wave, before encountering the minority (here ${ }^{3} \mathrm{He}$ ) cyclotron layer. In previous experiments performed in $\mathrm{H}$ plasmas varying the ${ }^{3} \mathrm{He}$ concentration it was shown that for inverted mode conversion scenarios the transition from ${ }^{3} \mathrm{He}$ minority heating (absorption at the minority cyclotron layer) to mode conversion heating occurs at a much lower ${ }^{3} \mathrm{He}$ concentration than in standard scenarios $\left(\sim 2 \%{ }^{3} \mathrm{He}\right.$ compared with around $10 \%$ in the standard scenarios) [30]. This was confirmed again in this set of experiments where at a ${ }^{3} \mathrm{He}$ concentration below $2 \%$, efficient ${ }^{3} \mathrm{He}$ minority heating was obtained. D ions from $\mathrm{D}$ beams and wall recycling and impurities, with a charge to mass ratio equal to that of deuterium, leads to a second mode conversion layer between the ${ }^{3} \mathrm{He}$ cyclotron resonance layer at the centre and the D cyclotron layer on the high-field side. The presence of multiple mode conversion layers and their effect on the heating efficiency as the ${ }^{3} \mathrm{He}$ concentration is increased, is described in detail in [31] and can be summarized as follows: below 2-2.5\% of ${ }^{3} \mathrm{He}$, efficient ${ }^{3} \mathrm{He}$ minority heating was observed, then the heating efficiency started to degrade and for ${ }^{3} \mathrm{He}$ concentrations between $4-6 \%$ it was difficult to couple power because one of the mode conversion layers then crossed the plasma boundary at the low-field side. At concentrations higher than $6 \%$ the heating efficiency was fully recovered. The power deposition, measured from the response of electron and ion temperatures caused by the modulation, have been reported in [31]. The recovered power was typically $70 \%$, dominated by electron heating, except for ${ }^{3} \mathrm{He}$ concentrations in the range $2-6 \%$.

The rotation profiles for a typical JET pulse are illustrated in figure 2. In this pulse the ${ }^{3} \mathrm{He}$ concentration was increased from $2 \%$ to $7 \%$, the magnetic field was $B_{0}=2.95 \mathrm{~T}$ and the plasma current $I_{\mathrm{p}}=1.5 \mathrm{MA}$ with an ICRF power of 3.6 MW. The cyclotron resonance of ${ }^{3} \mathrm{He}$ was located at the high-field side, at about $R \approx 2.73 \mathrm{~m}$, and the main mode conversion layer was located on the low-field side of the ${ }^{3} \mathrm{He}$ cyclotron resonance. In the outer region $3.4 \mathrm{~m}<R<$ $3.78 \mathrm{~m}$ there is almost no change in rotation and the plasma rotates with a nearly constant angular velocity of $0.5 \mathrm{krad} \mathrm{s}^{-1}$, which also agrees, within error bars, with the rotation before the application of ICRF power. This pulse shows a clear counter-rotation in the core, increasing with an increase of ${ }^{3} \mathrm{He}$ concentration.

There is a clear difference in the rotation profiles in the core at the instant of the first beam blip at $t=6.51 \mathrm{~s}$, and at beam blips later in the discharge. The rotation profiles at the second and third beam blip are remarkably similar. The coupled power, electron temperature and density profiles, measured with LIDAR are shown in figure $1(b)$. Ion temperature profiles are shown in figure 4. All are quite similar at the time of the beam blips. Thus, the factor affecting the rotation could be the change in the ${ }^{3} \mathrm{He}$ concentration. The ${ }^{3} \mathrm{He}$ concentration, which is measured in the divertor, varies little at the time of the beam blips. The signal shows a strong increase just before the beam blip. This, however, may not be relevant for the ${ }^{3} \mathrm{He}$ concentration in the plasma core at this first beam blip, because of the time required for the ${ }^{3} \mathrm{He}$ ions to penetrate to the core and for the plasma to be accelerated to a steady state. Note that at the last beam blip $(t=11.31 \mathrm{~s})$ the electron temperature was slightly lower due to a reduced ICRF power (as mentioned earlier and in [31] at ${ }^{3} \mathrm{He}$ concentrations between 4-6\% coupling was reduced as the mode conversion layer was crossing the plasma edge at the low-field side). At the same time the rotation profile shows a somewhat smaller central counter-rotation.

The influence of the deposition profile on rotation is illustrated in figures $3(a)-(e)$. In these figures we compare two discharges in the minority heating regime, \#79340 and $\# 79345$, with a low ${ }^{3} \mathrm{He}$ concentration varying from $0.5-1 \%$ for $\# 79340$ and from $0.5-1.5 \%$ for \#79345. The power deposition profiles differ due to the different magnetic fields; $B_{0}$ equal to 3.41 and $2.95 \mathrm{~T}$, respectively. In order to have similar $q$-profiles, the plasma currents were chosen to be $1.8 \mathrm{MA}$ and 1.5 MA, respectively. The difference in magnetic field displaced the cyclotron resonance of ${ }^{3} \mathrm{He}$ in $\# 79345$ over $40 \mathrm{~cm}$ towards the high-field side, compared with \#79340, resulting in a less central power deposition than in pulse \#79340. In order to compensate for the more off axis heating, more ICRH power was applied in \#79345, 4 MW, compared with 3.4 MW in \#79340. Due to more efficient heating, a higher electron temperature and density were obtained for \#79340. Time traces of the coupled ICRF power, NBI blips, central temperature and averaged density are shown in figure $3(a)$. The rotation profiles at $t=8.12 \mathrm{~s}, t=9.72 \mathrm{~s}$ and $t=11.31 \mathrm{~s}$ are shown in figure $3(c)$. Since the aim of the study is to clarify the cause of the rotation, we also give ion and electron temperatures and electron density profiles, here shown in figures $3(b),(d)$ and $(e)$. (The measurements at the first blip of \#79340 failed and have been left out). Although the power 

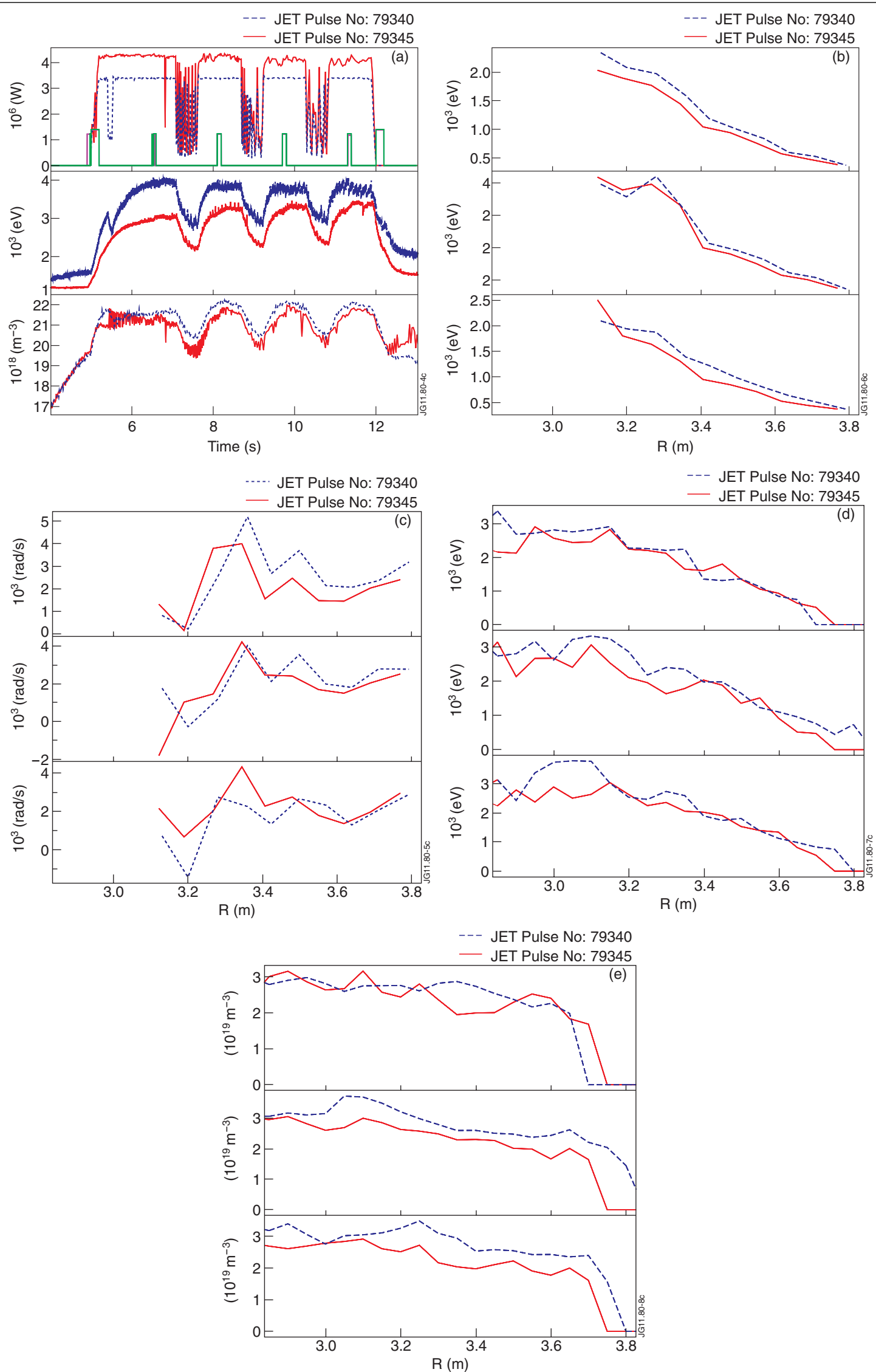

Figure 3. A comparison between the discharges \#79340 (blue dashed) and \#79345 (red full line) both with a concentration of ${ }^{3} \mathrm{He}<1.5 \%$. Shown are (a) ICRH and NBI power (top graph), electron temperature (middle graph) at $R=2.95 \mathrm{~m}$, volume-averaged density (bottom graph); (b) electron temperature profiles, $(c)$ angular rotation profiles. The confidence interval is $\pm 0.5 \mathrm{krad} \mathrm{s}^{-1}$ in the outer part and $\pm 1.5 \mathrm{krad} \mathrm{s}^{-1}$ in the core, $(d)$ ion temperature profiles, $(e)$ density profiles measured with LIDAR. In figures $3(b)-(e)$ the top graph corresponds to $t=8.12 \mathrm{~s}$, the middle graph to $t=9.72 \mathrm{~s}$ and the bottom graph to $t=11.31 \mathrm{~s}$. 

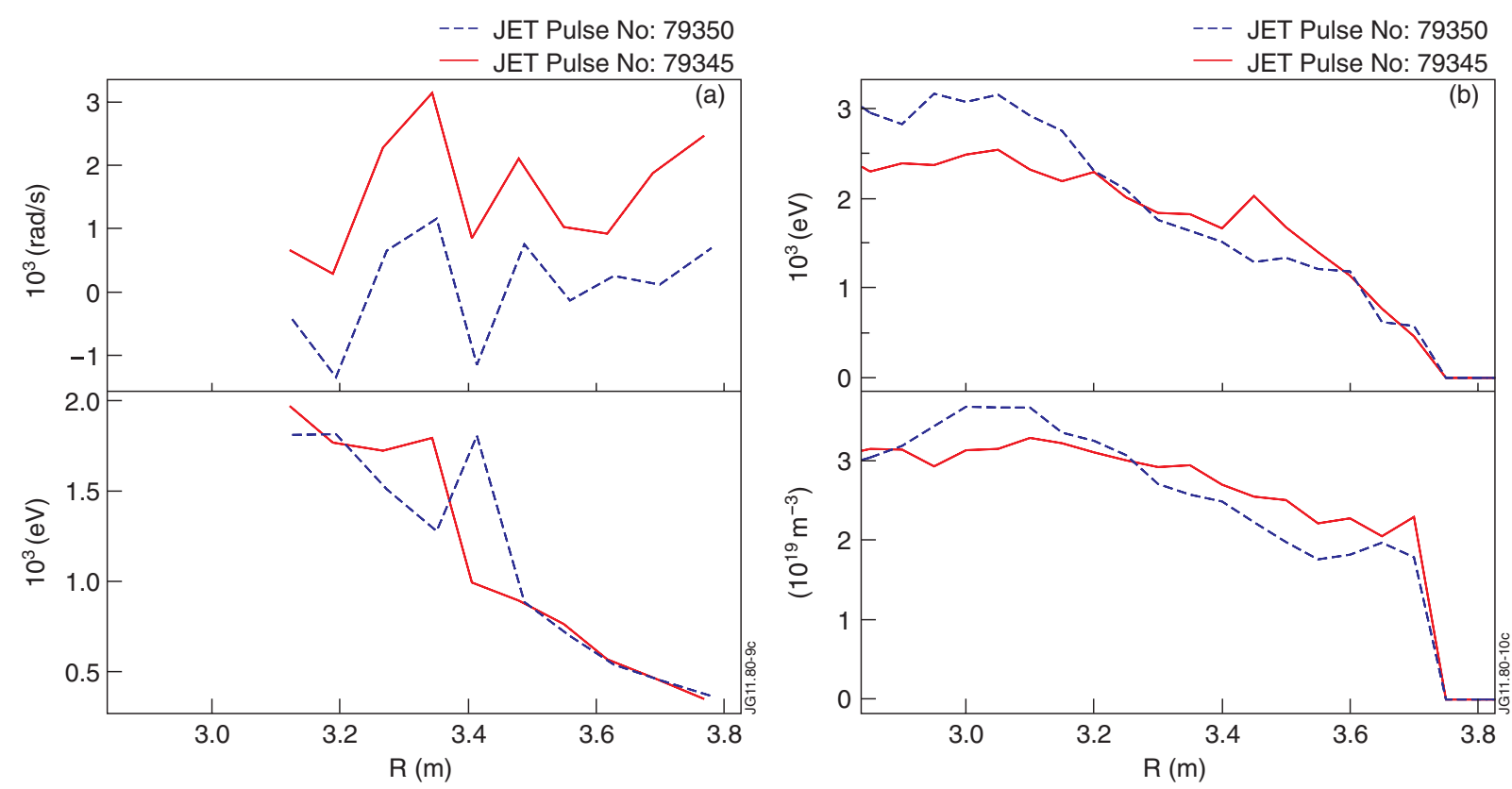

Figure 4. A comparison between the various plasma profiles of discharges \#79345 with ${ }^{3} \mathrm{He}<1 \%$ (red full line) and \#79350 with ${ }^{3} \mathrm{He} \approx 6 \%$ (blue dashed) at $t=6.51 \mathrm{~s}$. (a) Angular rotation (top graph), the confidence interval is $\pm 0.5 \mathrm{krad} \mathrm{s}^{-1}$ in the outer part and $\pm 1.5 \mathrm{krad} \mathrm{s} \mathrm{s}^{-1}$ in the core, and ion temperature (bottom graph) $(b)$ electron temperature (top graph) and electron density (bottom graph) both measured with LIDAR.

deposition profiles are different, the rotation profiles were quite similar within error bars of the order $50 \%$. In the outer region the plasma rotates with a nearly constant angular rotation at around $2 \mathrm{krad} \mathrm{s}^{-1}$, having a tendency to decrease towards the core. Experiments with $\mathrm{H}$-minority heating have revealed that the counter-rotation in the centre decreases with the plasma current [3]. However, there was a large spread in these data, indicating that other variables were important for the rotation. For the standard mode conversion, the magnitude of the core counter-rotation was found to increase with power [32]. However, these discharges were performed with $-90^{\circ}$ phasing (relative phasing of the four straps $0,-\pi / 2,-\pi,-3 \pi / 2$ ) also including a net momentum, transferred to the plasma from the wave. The similarity of the rotation profiles in figure $3(c)$ suggests that a change in rotation, due to the change in plasma current, is compensated by a change in the electron temperature or ICRF power. All these changes differ by $20 \%$ for the two discharges.

In figure 4 , we compare the rotation profile at the beam blip, occurring at $t=6.51 \mathrm{~s}$, for discharges \#79350 and \#79345. The rotation profile at this first blip differed from the rotation profile at later beam blips. The ${ }^{3} \mathrm{He}$ concentration was below $1 \%$ for \#79345. The two discharges have the same magnetic field and plasma current but different heating powers, the electron temperature and line-averaged density; e.g. \#79350 was heated with an ICRH power of 3.6 MW compared with \#79345 with $4 \mathrm{MW}$, the electron temperature at the magnetic axis was $3.2 \mathrm{keV}$ compared with $3.0 \mathrm{keV}$ (see figures $1(a)$ and $3(a))$ and the line-averaged densities were $2.2 \times 10^{19} \mathrm{~m}^{-2}$ compared with $2.0 \times 10^{19} \mathrm{~m}^{-2}$. The rotation in the outer part is lower for \#79350 than \#79345. The difference between the rotation profiles is almost constant and equal to $2 \mathrm{krad} \mathrm{s}^{-1}$ except at the innermost measurement points. The agreement between the rotation profiles (except for a certain offset) suggests that for \#79350, the ${ }^{3} \mathrm{He}$ concentration in the core at the first beam blip is lower than what the measurements, deduced from the divertor light, gives, about $6 \%$, because of the increase in the ${ }^{3} \mathrm{He}$ concentration preceding the measurements (this discrepancy was discussed in detail in [27]) or the plasma had not had time enough to be close to a steady state. Assuming that the torque depends on electron heat transport by drift waves, the stronger torque, caused by the higher electron temperature in \#79345, could be compensated by the higher density to give the same rotation. This suggests that the rotation is correlated with the electron temperature rather than with the ICRF power.

Next we compare the rotation at higher ${ }^{3} \mathrm{He}$ concentrations for the discharges \#79350 and \#79353 that had different positions for the mode conversion layer, due to differences in the magnetic field. In discharge \#79350, $B_{0}=2.95 \mathrm{~T}$ and $I_{\mathrm{p}}=1.5 \mathrm{MA}$ and the ICRF power was about $3.6 \mathrm{MW}$ at the first three points and 1.4 MW at the last. The ${ }^{3} \mathrm{He}$ concentration increased from 2-7\%. In discharge \#79353, $B_{0}=3.41 \mathrm{~T}$ and $I_{\mathrm{p}}=1.8 \mathrm{MA}$. The ${ }^{3} \mathrm{He}$ concentration increased from $6-15 \%$. The coupled ICRF power, NBI blips, central temperature and averaged density are shown in figure $5(a)$. The ICRF power varied, as shown in figure 5(a), and during the beam blips it was $2.4,2.5,3.0$ and $3.5 \mathrm{MW}$. The rotation profiles at $t=6.53 \mathrm{~s}$, $t=8.12 \mathrm{~s}, t=9.72 \mathrm{~s}$ and $t=11.31 \mathrm{~s}$, are shown in figure $5(b)$. Figure $5(c)$ shows the power deposition profile for direct electron heating at $t=8.9 \mathrm{~s}$ for $\# 79350\left({ }^{3} \mathrm{He}\right.$ concentration of $6 \%$ ) and at $t=8.7 \mathrm{~s}$ for \#79353 $\left({ }^{3} \mathrm{He}\right.$ concentration of $8 \%$ ). The power deposition profiles of discharge \#79350 were more peaked in the centre, compared with \#79353. Later in the discharge, the ${ }^{3} \mathrm{He}$ concentration increased, and the peak of the power deposition profile in \#79353 was displaced slightly, further outwards. Depending on the method of calculating the power, between $66-78 \%$ of the coupled power could be 

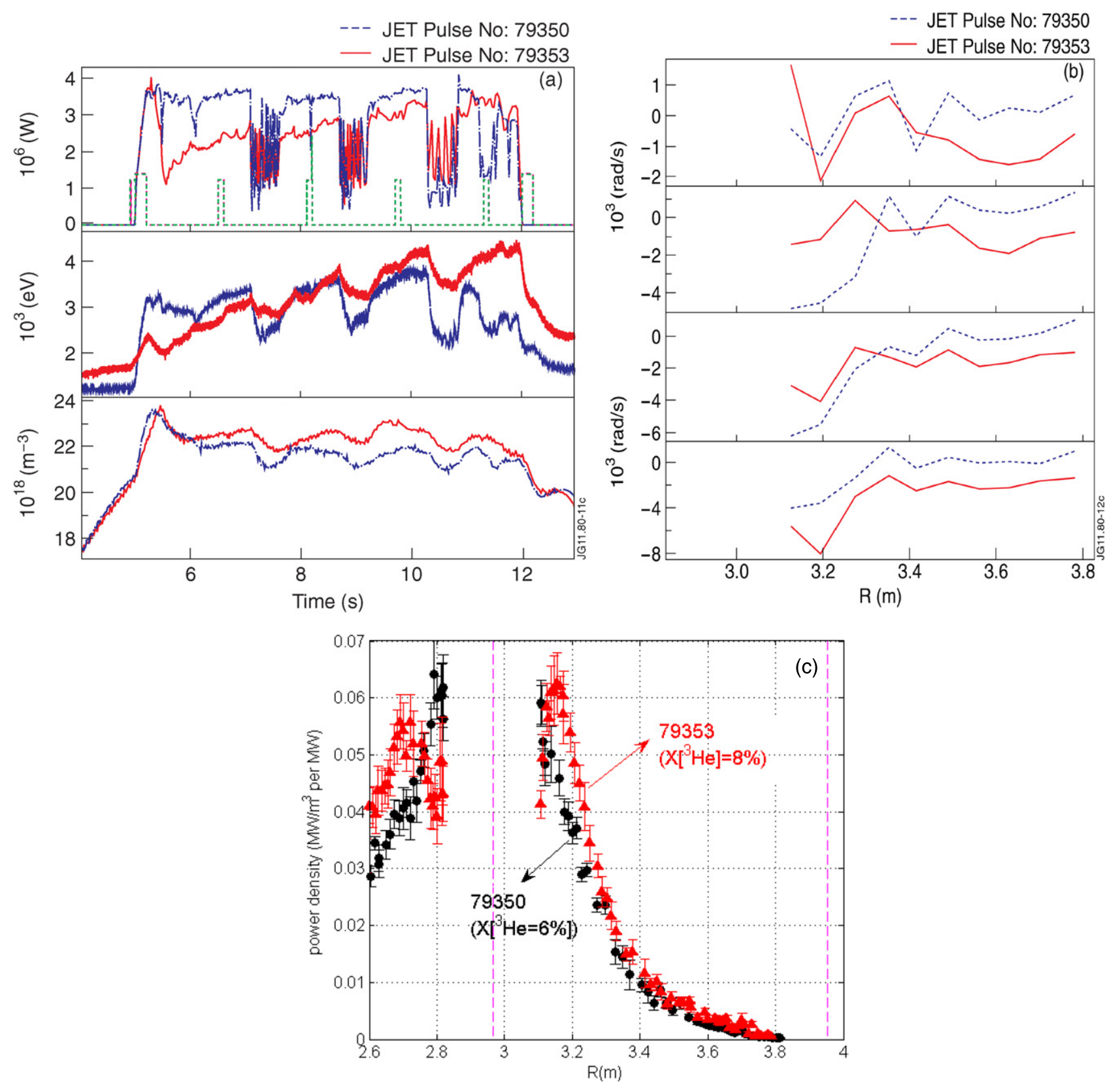

Figure 5. A comparison between the discharges \#79350 (blue dashed line) and \#79353 (red full line) (a) ICRH and NBI power (top panel), electron temperature (middle panel) at $R=2.95 \mathrm{~m}$, line-averaged density (bottom panel). (b) Angular rotation profiles at different times from top to bottom at $t=6.51 \mathrm{~s}, t=8.12 \mathrm{~s}, t=9.72 \mathrm{~s}$ and $t=11.31 \mathrm{~s}$. The confidence interval is $\pm 0.5 \mathrm{krad} \mathrm{s}^{-1}$ in the outer part and $\pm 1.5 \mathrm{krad} \mathrm{s}^{-1}$ in the core for the lower values and $\pm 2 \mathrm{krad} \mathrm{s}^{-1}$ for the higher. (c) The power deposition profile normalized to coupled power of direct electron heating obtained by modulation of RF-power for discharges for \#79350 (black) and \#79353 (red) between $t=8.7 \mathrm{~s}$ and $8.9 \mathrm{~s}$, with ${ }^{3} \mathrm{He}$ concentrations of $6 \%$ and $8 \%$, respectively.

accounted for in pulse \#79350 using modulation and between 82-90\% in pulse \#79353. However, the slightly lower power accounted for in \#79350 (which had the most peaked power deposition profile) was most likely due to a lack of temperature measurements in the plasma centre. One would therefore expect that heating efficiency, a fraction of the absorbed power over the total power within the error bars, to be similar for the two discharges.

At the two first beam blips, the ICRF power was lower for \#79353, but the temperatures and line-averaged densities were similar. The angular velocity in the outer region of the plasma in pulse \#79353 is nearly constant and does not vary for the different beam blips as the ${ }^{3} \mathrm{He}$ concentration changes. Furthermore, the angular velocity was also similar to that in the ohmic phase. The rotation was about $1 \mathrm{krad} \mathrm{s}^{-1}$ lower than for discharge \#79350. At the first beam blip, the rotation profiles were similar except for this offset. It should be noted that the discharges were done in two different sessions. The rotation at the outer part of the plasma has been seen to vary between sessions.

A significant counter-rotation in the core was seen for $\# 79350$ at $t=8.12 \mathrm{~s}$, at $t=9.72 \mathrm{~s}$ and $t=11.31 \mathrm{~s}$ and 
for $\# 79353 t=9.72 \mathrm{~s}$ and $t=11.31 \mathrm{~s}$. For \#79350 at $t=11.31 \mathrm{~s}$ the ICRF power tripped just before the start of the NBI heating and the electron temperature varied; therefore, this point in the comparison is excluded. When comparing the rotation for $\# 79350$ at $t=8.12$ and $t=9.72 \mathrm{~s}$ we note that the powers and the line-averaged densities were the same but the electron temperature and the rotation were both lower at $t=8.12 \mathrm{~s}$, indicating a scaling with electron temperature instead of power. Then we compare \#79350 at $t=9.72 \mathrm{~s}$ with $\# 79353$ at $t=11.31 \mathrm{~s}$ for which the differences in counter-rotation and the electron temperatures were similar. In \#79353 the plasma current was $20 \%$ higher, the density $5 \%$ higher and the power $10 \%$ lower with a less peaked power profile, indicating a better confinement with higher plasma current. Assuming a scaling of the rotation inversely with plasma current, one would then expect the counter-rotation to be higher in \#79350 than in \#79353. If power is more relevant than electron temperature for scaling, an even larger counterrotation would then be expected in \#79350. The difference in rotation from scaling with respect to plasma current, electron temperature or power could be an effect of mode conversion or position of the heating, but is within the error bars and can thus not be taken as conclusive evidence of an effect of mode conversion on the rotation. We then compare the rotation for $\# 79353$ at $t=9.72 \mathrm{~s}$ and $t=11.31 \mathrm{~s}$. For the counterrotation, the power and the electron temperature were lower at $t=9.72 \mathrm{~s}$, indicating a scaling of the rotation with either the electron temperature or the power.

\subsection{Second harmonic heating of ${ }^{3} \mathrm{He}$ in H plasmas}

Another set of experiments was performed in order to investigate the heating efficiency of the second harmonic heating of ${ }^{3} \mathrm{He}$ in $\mathrm{H}$ plasmas, a scenario investigated for the non-activated phase of ITER at half field [29]. For this study, these plasmas were very interesting as it was demonstrated that up to $15 \%$ of ${ }^{3} \mathrm{He}$, the dominant absorption mechanism was direct electron heating via TTMP/ELD. The influence of the ${ }^{3} \mathrm{He}$ concentration in these $\mathrm{H}$ plasmas is illustrated in figure 6. The rotation was measured at $t=11.92 \mathrm{~s}$ in three pulses with $I_{\mathrm{p}}=1.4 \mathrm{MA}$ and $B_{0}=2.65 \mathrm{~T}$. The three discharges had nearly the same ICRH power, ion temperature and diamagnetic energy. Discharge \#79361 had a ${ }^{3} \mathrm{He}$ concentration of $17 \%$ with an ICRF power of 5.2 MW; discharge \#79362 had a ${ }^{3} \mathrm{He}$ concentration of $11 \%$ with an ICRF power of $5.5 \mathrm{MW}$; and discharge \#79363 had a ${ }^{3} \mathrm{He}$ concentration of $8 \%$ with $5.5 \mathrm{MW}$. The fraction of the ICRF power absorbed by electrons and ions depends on the ${ }^{3} \mathrm{He}$ concentration. At the highest ${ }^{3} \mathrm{He}$ concentration (e.g. in discharge \#79361) the ion absorption was comparable to the electron absorption. At the lowest concentration (e.g. discharge \#79363) electron heating by TTMP/ELD dominates, which is consistent with the higher electron temperature obtained in such discharges. The heating efficiencies for these discharges were poor because of weak single pass damping due to the unfavourable polarization and relatively long wavelengths of the magnetosonic waves. The measured heating efficiencies at an earlier time were in the range $25-30 \%$ [29], about half or less than that of the standard

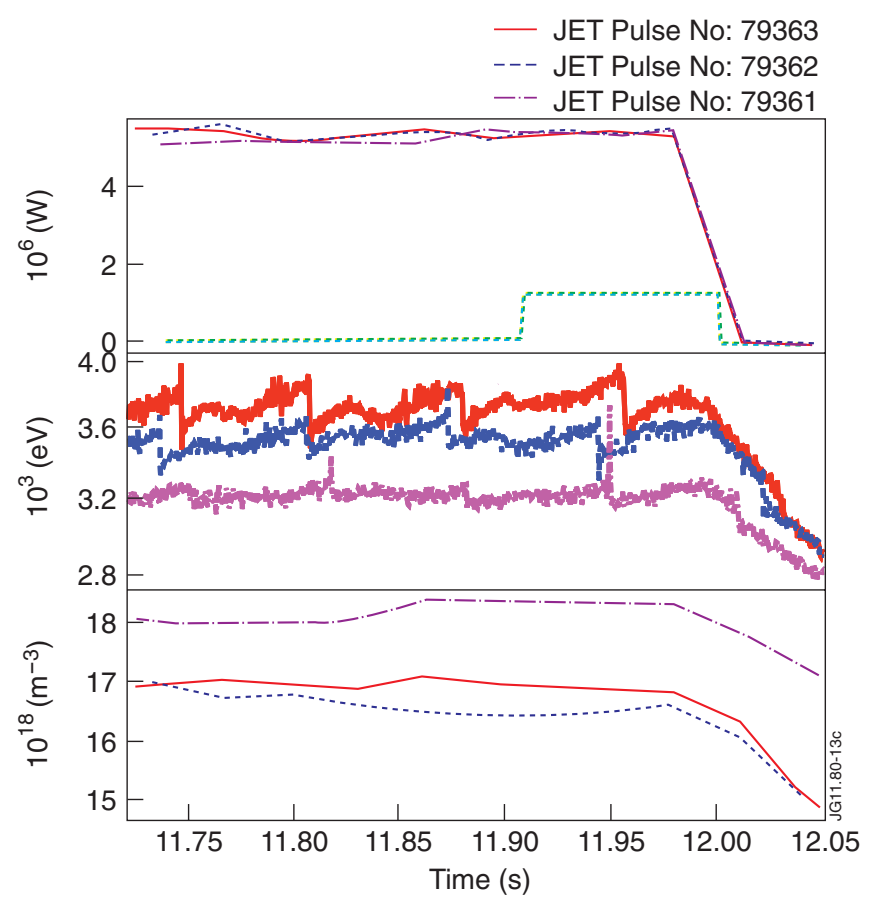

Figure 6. A comparisons between the discharges with $\omega=2 \omega_{\mathrm{c} 3} \mathrm{He}$ \#79361 with $17 \%{ }^{3} \mathrm{He}$ (red full line), \#79362 with $11 \%{ }^{3} \mathrm{He}$ (blue dashed line ) and \#79363 with $8 \%{ }^{3} \mathrm{He}$ (magenta dashed dotted line). Time traces of the discharges: ICRH and NBI power (top graph), electron temperature (middle graph) at $R=2.94 \mathrm{~m}$ measured with ECE radiometer, volume-averaged electron density (bottom graph).

and the inverted mode conversion scenarios. The electron temperatures, the rotation and the plasma currents were about the same as for pulse \#79350, with an inverted mode conversion heating. The density was about $20 \%$ lower. Despite the lower heating efficiencies, these discharges showed a rather large counter-rotation in the core, as shown in figure 8 for discharge \#79363. The discharge with the lowest counter-rotation was \#79361 having the lowest electron temperature, but also the highest density.

\subsection{Comparisons of the rotation for different ICRH scenarios}

Common to many of the rotation profiles seen during ICRH is the nearly constant angular rotation in the outer part of the plasma, between $3.4 \mathrm{~m}<R<3.7 \mathrm{~m}$. When reliable data exist for the ohmic phase, before or after applying the ICRF heating, the change in rotation in the outer part is rather small. The rotation in the outer part varies between $0-5 \mathrm{krad} \mathrm{s}^{-1}$ for different discharges, and is typically constant during several discharges or throughout the session, but can vary significantly between sessions. It is possible that the rotation in this region is sensitive to the magnetic field geometry in the scrape off layer. The outer part of the plasma is, in general, co-rotating except for a large magnetic field ripple [8] and some $\mathrm{H}$-mode plasmas $[2,24]$.

The recently reported rotation in the standard mode conversion scenario, with ${ }^{3} \mathrm{He}$ in deuterium plasmas in JET [31], demonstrates a similar counter-rotation in the core as the inverted mode conversion scenario. The magnitude of the core rotation increases with ICRH power and decreases 

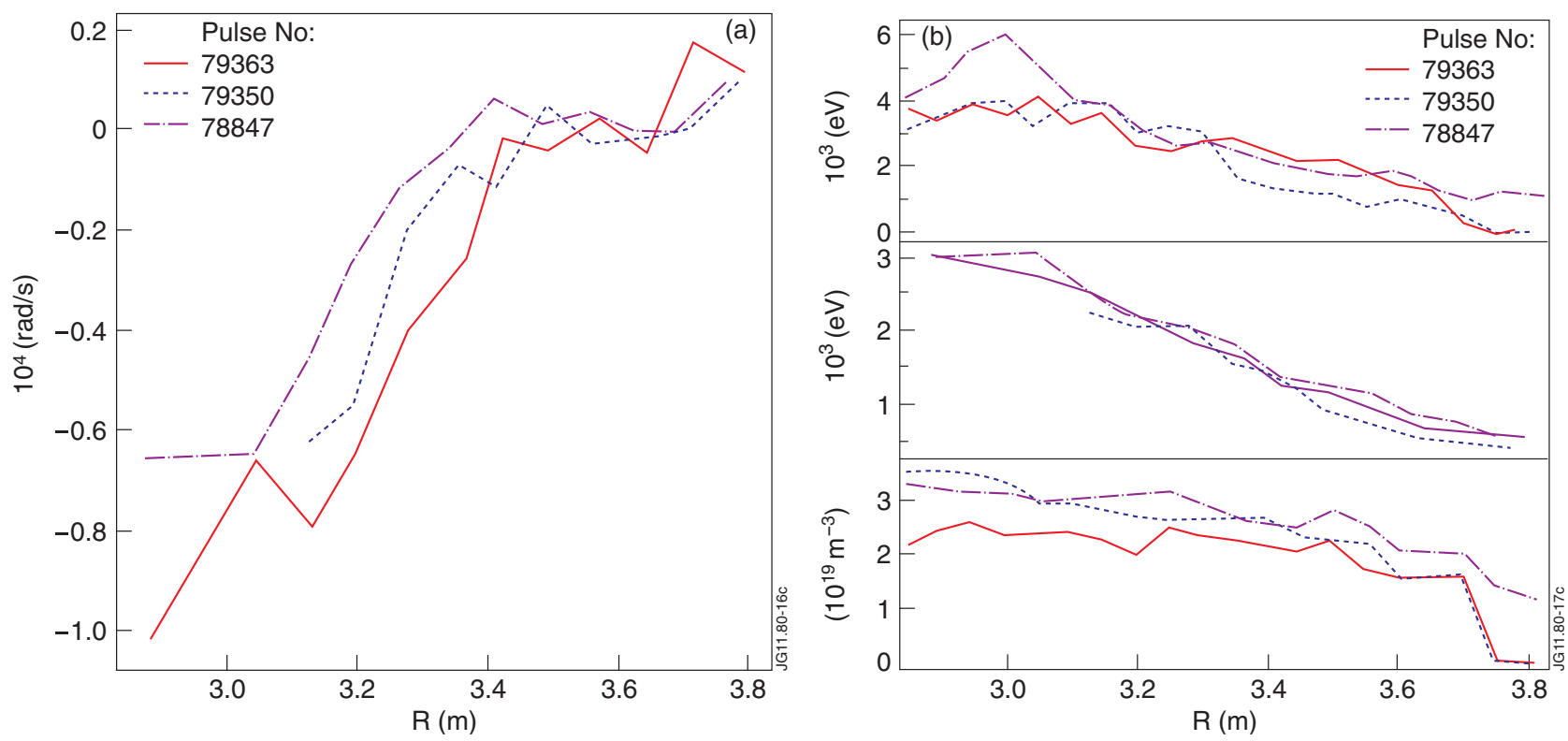

Figure 7. A comparison of the rotation profiles obtained with different heating scenarios: $\omega=2 \omega_{\mathrm{c} 3 \mathrm{He}}(\# 79363$ red full line) at $t=11.91 \mathrm{~s}$; an inverted mode conversion at a high ${ }^{3} \mathrm{He}$ concentration (\#79350 blue dashed line) at $t=9.72 \mathrm{~s}$; a standard mode conversion with a high concentration and $3 \mathrm{MW}$ of ICRF power and $-90^{\circ}$ (i.e. $0-\pi / 2-\pi-3 \pi / 2$ ) phasing of the four antenna straps (\#78847 magenta dashed dotted) at $t=7.01 \mathrm{~s} ;(a)$ the rotation profiles, the confidence interval is $\pm 0.5 \mathrm{krad} \mathrm{s}^{-1}$ in the outer part and $\pm 2 \mathrm{krad} \mathrm{s}^{-1}$ in the core, and (b) the electron temperature (top graph), ion temperature (middle graph) and density (bottom graph).
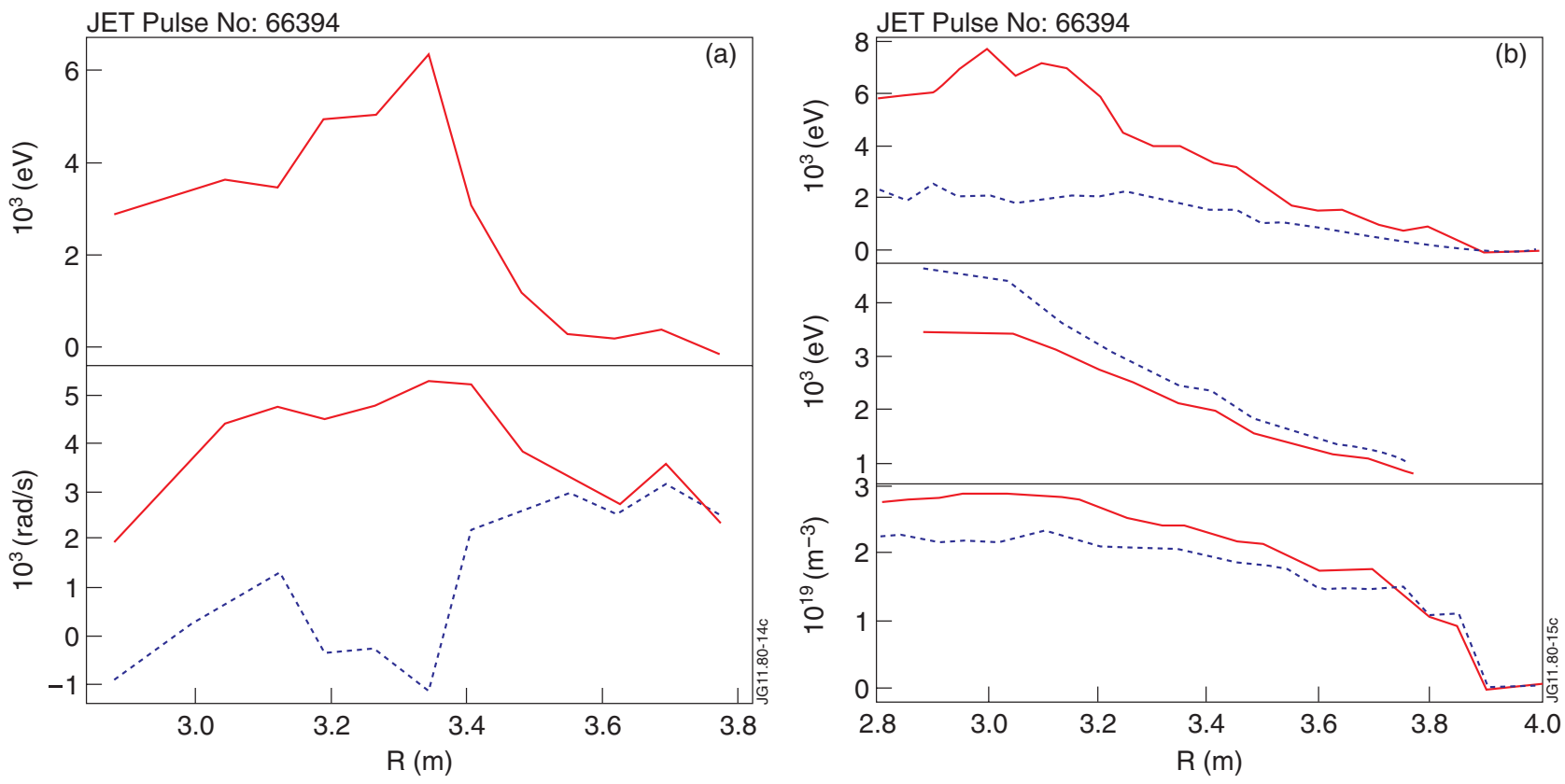

Figure 8. The rotation profiles for \#66394 with $4.8 \mathrm{MW}$ hydrogen minority heating $I_{\mathrm{p}}=2.4 \mathrm{MA}, B_{0}=2.4 \mathrm{~T}, f=42 \mathrm{MHz}$, $n_{\mathrm{H}} / n_{\mathrm{D}}=0.03$, off axis heating on the high-field side. ( $a$ ) The difference between the rotation profiles at $t=7.03 \mathrm{~s}$ and $t=11.53 \mathrm{~s}$ (top graph), rotation profile at $t=7.03 \mathrm{~s}$ during the ohmic heating phase (bottom graph, red full line) and at $t=11.53 \mathrm{~s}$ (blue dashed line). (b) Electron temperature (top graph), ion temperature (middle graph) and density profile measured with LIDAR (bottom graph).

with the plasma current. The rotation in the plasmas heated with waves, at the frequency of the second harmonic cyclotron resonance of ${ }^{3} \mathrm{He}$ in hydrogen plasmas, demonstrates similar counter-rotation in the core. In figure 7 a comparison of the rotation profiles for different heating scenarios with dominating electron heating, is shown. For discharge \#78847 with a standard mode conversion, the launched ICRF power was about half and the plasma current about twice that of the second harmonic ${ }^{3} \mathrm{He}$ in $\mathrm{H}$ of \#79363. The heating in the standard mode conversion scenario $\left({ }^{3} \mathrm{He}\right.$ in D-plasmas) was conducted with $-90^{\circ}$ (i.e. $0,-\pi / 2,-\pi,-3 \pi / 2$ ) phasing of the antenna straps, in order to increase the counter-rotation by the wave momentum [31]. Although the rotation profiles are similar, they have different plasma currents, ICRF power, relative strap phasings and heating efficiencies. Therefore one cannot draw any conclusions as to whether the effects 
associated with mode conversion contribute to the rotation or not. However, for the discharges with the inverted mode conversion heating scheme there was no conclusive evidence that effects associated with mode conversion contributed to the rotation.

There is a noticeable difference in rotation between heating scenarios involving direct absorption of the wave power by ions and those with direct absorption on the electrons, even though both may result in electron heating. This difference may be caused by orbit effects of fast ions. This is illustrated by a comparison between the rotation profiles presented in sections 3.1 and 3.2 and the rotation profiles for the most common heating scenario in JET, H-minority in deuterium, which at low concentrations or high powers gives rise to electron heating through fast ions. At low plasma currents, typically below $2 \mathrm{MA}$, the rotation profile in the core is hollow for H-minority heating in D-plasmas [3]. Compared with the value at the boundary, the core rotates in the countercurrent direction. At higher currents, equal or above 2.4 MA, the core rotates in the co-current direction, sometimes weakly hollow near the magnetic axis. The counter-rotation increases in the core for waves launched in the counter-current direction, such as for $-90^{\circ}$ phasing (relative phasing of the four straps $0-\pi / 2-\pi-3 \pi / 2$ ), which is consistent with absorption of the momentum of the wave $[3,13]$. The rotation profile for dipole heating is shown in figure 8 at $I_{\mathrm{p}}=2.4 \mathrm{MA}$, $B_{0}=2.4 \mathrm{~T}$, ICRF frequency $f=42 \mathrm{MHz}, \mathrm{H}$ concentration $n_{\mathrm{H}} / n_{\mathrm{D}}=0.03$, and off axis heating with the cyclotron resonance intersecting the midplane at about $r=0.4 \mathrm{~m}$ on the high-field side. Figure 8 also illustrates the difficulties to scale the core rotation: to obtain rotation profiles like the ones illustrated in figure 8(a) requires spatially alternating torques consistent with momentum transport from one region to another. Note also the increase in the co-rotation in the region between $3.35 \mathrm{~m}<R<3.55 \mathrm{~m}$, a phenomenon that was absent in experiments with direct electron heating. The small difference in rotation between the ohmic and the ICRFphase suggests that the rotation in the outer region is not an ICRF-effect.

\section{Conclusions and discussions}

A number of rather weak mechanisms seem to give rise to a toroidal rotation of tokamak plasmas as they are heated with ICRF waves, and this makes it difficult to scale the rotation to future experiments. Some of them are intrinsic depending on transport of momentum caused by heating. Others are specific to the heating scenario used, caused by the finite wave momentum absorbed by electrons and ions or finite orbit width effects of fast ions. Absorption of wave momentum, non-ambipolar transport by orbit effects and ripple had earlier been identified, and experimentally confirmed, as mechanisms causing the ICRF heated plasma to rotate toroidally. However, there are changes in the rotation profiles, for which a reason has not yet been identified. In order to separate effects caused by wide ion orbits from effects caused by heating and transport, toroidal plasma rotation was studied for scenarios with waves directly damped on the electrons. Two heating scenarios in $\left({ }^{3} \mathrm{He}\right)-\mathrm{H}$ plasmas are used in this study: (i) inverted mode conversion for which the transition from ion minority heating to mode conversion heating takes place at lower ${ }^{3} \mathrm{He}$ concentrations than in the standard mode conversion scenarios and (ii) heating at the second harmonic ${ }^{3} \mathrm{He}$ resonance in a hydrogen plasmas. For the inverted mode conversion scenario, the mode converted slow wave is damped by ELD at higher minority concentrations. For heating at the second harmonic ${ }^{3} \mathrm{He}$ resonance in hydrogen plasmas, with the parameters used in these experiments, the dominating absorption mechanism of the fast wave is damping on electrons by TTMP/ELD without involving the mode conversion. To minimize the effect of momentum absorption by a directed wave spectrum, the experiments had been carried out with dipole (i.e. $0 \pi 0 \pi$ ) phasing, which gives almost toroidal symmetric wave spectra with little net toroidal momentum. The experiments were carried out with the standard low field ripple $(\delta=0.08 \%)$ to avoid rotational effects caused by the ripple, which has earlier been found to give rise to counter-rotation [8].

In the outer part of the plasma, between $3.4 \mathrm{~m}<R<$ $3.75 \mathrm{~m}$, the plasma 'rotates' with an almost constant angular velocity in the co-current direction for these experiments in L-mode plasmas. The rotation in this region could vary significantly between different experimental sessions with minor changes within the sessions, even when the minority concentration or the heating scenario changed. The rotation in ohmic plasmas exhibits similar rotation profiles, but with an overall lower rotation in the core [3]. When reliable measurements existed for the rotation in the ohmic phase either before or after the application of RF power, the rotation in the outer part of the plasma was rather similar. Clear systematic changes in the rotation in this region have only been seen with increased ripple in JET [8].

In the core the plasma rotated in the counter-current direction for these L-mode plasmas. The rotation profiles for heating at the second harmonic ${ }^{3} \mathrm{He}$ resonance in $\mathrm{H}$ plasmas demonstrated similar rotation profiles as in the inverted mode conversion experiments. Counter-rotation in the core has earlier been seen during standard mode conversion heating with ${ }^{3} \mathrm{He}$ in D-plasmas. Thus, for these three heating methods, for which the waves were absorbed directly on electrons, and fast ion effects were negligible, the rotation profiles were similar with the counter-rotation in the core, scaling inversely with the plasma current. Counter-rotation in the core has also been seen for minority heating in JET at a low plasma current [3]. Note that this scaling does not contradict the earlier reported scaling at JET [3], which indicates that the rotation increases with the plasma current, since at a low plasma current the rotation was negative, i.e. counter-rotating, and increasing in magnitude as the current decreased. Furthermore, when taking into account the changes in the plasma current, the experiments reported here demonstrated a somewhat better correlation with the temperature profile than with ICRH power, suggesting that the dominating mechanism for a counterrotation in the core is related to the electron heat transport rather than being a phenomenon directly related to the ICRF heating itself. Since the changes within the error bars in the rotation for the direct electron heating scenarios seem to be 
consistent with changes in the plasma current, the electron temperature and density, and since the rotation profiles for heating at the second harmonic heating of ${ }^{3} \mathrm{He}$ were similar to the discharges reported here with an inverted mode conversion, there is no conclusive evidence that mode conversion in itself affects rotation for these discharges. This does not necessarily contradict earlier results on Alcator C-Mod, where enhanced rotation was found in a narrow parameter region, and that the effects associated with mode conversion could have contributed to the rotation in experiments at JET with standard mode conversion [30]. It should be kept in mind that because of the relatively large errors of the rotation measurements at these low velocities, it is difficult to draw quantitative conclusions on the scaling, because the small variations observed are almost within the capabilities of the diagnostic.

The observed counter-rotation in the core surrounded by a nearly rigid rotation in the outer part of the plasma, or even absence of rotation, suggests a dipolar-like torque in the core. Such a torque is expected to arise both from wave-particle interaction e.g. by drift waves in anomalous transport, where momentum is taken from one part of the plasma and given to another part of the plasma [22,23]. Similar behaviour of the rotation has been reported in other machines as well, in ohmic discharges [14-16] and in discharges heated with other methods to heat electrons, such as ECRH in TCV [17-19], as discussed in the introduction.

There is a clear difference between the difference in rotation at the core and outer region of the plasma as the concentration of ${ }^{3} \mathrm{He}$ ions changes for the inverted mode conversion experiments. The difference is lowest at the lowest concentration of the minority species, when the ions are heated by cyclotron damping and high-energy tails are created on the ion distribution function, and the power is transferred to the electrons by collisions. A possible explanation is that in addition to the rotation caused by electron heating the rotation is influenced by ion orbit effects. Orbit effects on the rotation have earlier been identified to give a dipolar torque in JET [3].

(C) Euratom 2012.

\section{References}

[1] Eriksson L-G et al 1992 Plasma Phys. Control. Fusion 34863

[2] Eriksson L-G et al 1997 Plasma Phys. Control. Fusion 3927

[3] Eriksson L-G et al 2009 Plasma Phys. Control. Fusion 51044008

[4] Rice J E et al 2004 Nucl. Fusion 44379

[5] Eriksson L-G et al 2001 Nucl. Fusion 4191

[6] Assas S et al 2007 Radio Frequency Power in Plasmas ed P M Ryan and D A Rasmunsen AIP Conf. Proc. 933103 (New York: Melville)

[7] Noterdaeme J M et al 2003 Nucl. Fusion 43274

[8] Nave M F F et al 2010 Phys. Rev. Lett. 105105005

[9] Lin Y et al 2008 Phys. Rev. Lett. 101235002

[10] Rice J E et al 1998 Nucl. Fusion 3875

[11] Rice J E et al 1999 Nucl. Fusion 391175

[12] Rice J E et al 2001 Nucl. Fusion 41277

[13] Eriksson L-G et al 2004 Phys. Rev. Lett. 92235001

[14] Hutchinson I H et al 2000 Phys. Rev. Lett. 843330

[15] Scarabosio A et al 2006 Plasma Phys. Control. Fusion 48663

[16] Bortolon A et al 2006 Phys. Rev. Lett. 97235003

[17] Duval B P et al 2007 Plasma Phys. Control. Fusion 49 B195

[18] Camenen Y et al 2010 Plasma Phys. Control. Fusion 52124037

[19] Angioni C et al 2011 Phys. Rev. Lett. 107215003

[20] Hedin J et al 1998 Proc. Joint Varenna- Lausanne Workshop 'Theory of Fusion plasmas' (Varenna, Italy) p 467, ISBN 88-7794-167-7

[21] Hellsten T et al 2004 Nucl. Fusion 44892

[22] Hellsten T 2009 Radio Frequency Power in Plasmas ed V Bobokov and J-M Noterdaeme AIP Conf. Proc. 1187 625 (New York: Melville)

[23] Hellsten T 2011 Plasma Phys. Control. Fusion 53054007

[24] Nave M F F et al 2012 Plasma Phys. Control. Fusion 54074006

[25] Greenwald M et al 1997 Nucl. Fusion 37793

[26] Mantsinen M J et al 2004 Nucl. Fusion 4433

[27] Van Eester D et al 2009 Plasma Phys. Control. Fusion 51044007

[28] de la Luna E 2004 Rev. Sci. Instrum. 753831

[29] Lerche E et al 2011 Plasma Phys. Control. Fusion 53124019

[30] Mayoral M-L et al 2006 Nucl. Fusion 46 S550

[31] Van Eester D et al Plasma Phys. Control. Fusion 54074009

[32] Lin Y et al Plasma Phys. Control. Fusion 54074001 